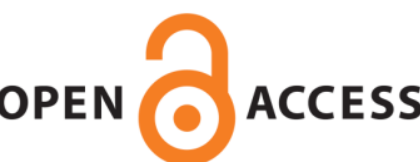

OPEN ACCESS

UWS Academic Portal

\title{
A comparison of physical activity from Actigraph GT3X+ accelerometers worn on the dominant and non-dominant wrist
}

Buchan, Duncan; McSeveney, Fiona; McLellan, Gillian

Published in:

Clinical Physiology and Functional Imaging

DOI:

10.1111/cpf.12538

Published: 31/01/2019

Document Version

Peer reviewed version

Link to publication on the UWS Academic Portal

Citation for published version (APA):

Buchan, D., McSeveney, F., \& McLellan, G. (2019). A comparison of physical activity from Actigraph GT3X+ accelerometers worn on the dominant and non-dominant wrist. Clinical Physiology and Functional Imaging, 39(1), 51-56. https://doi.org/10.1111/cpf.12538

\section{General rights}

Copyright and moral rights for the publications made accessible in the UWS Academic Portal are retained by the authors and/or other copyright owners and it is a condition of accessing publications that users recognise and abide by the legal requirements associated with these rights.

Take down policy

If you believe that this document breaches copyright please contact pure@uws.ac.uk providing details, and we will remove access to the work immediately and investigate your claim. 
A comparison of physical activity from Actigraph GT3X+ accelerometers worn on the dominant and non-dominant wrist

Duncan S. Buchan*, Fiona McSeveney and Gillian McLellan.

Institute of Clinical Exercise and Health Science, The University of the West of Scotland, Hamilton, United Kingdom, ML3 0JB.

* Corresponding author

Email: duncan.buchan@uws.ac.uk

Dr Duncan Buchan

Senior Lecturer in Exercise Science

Institute of Clinical Exercise and Health Science

School of Science and Sport

University of the West of Scotland

Almada Street

Hamilton (Room A430)

South Lanarkshire

ML3 0JB

Short title: Comparing wrist accelerometers

Word count: 3464

Summary word count: 250

Number of tables: 2

Number of figures: 1 
Summary:

The purpose of this study was to evaluate the agreement between several activity measures using raw acceleration data from accelerometers worn concurrently on the dominant and non-dominant wrist. Fifty-five adults (31.9 \pm 9.7 years, 26 males) wore two ActiGraph GT3X+ monitors continuously for 1 day, one on their non-dominant wrist and the other on their dominant wrist. Paired t-tests were undertaken with sequential Holm-Bonferroni corrections to compare wear time, moderate-vigorous physical activity (MVPA), time spent in 10-min bouts of MVPA (MVPA $10 \mathrm{~min})$ and the average magnitude of dynamic wrist acceleration (ENMO). Level of agreement between outcome variables from the wrists were examined using Intraclass Correlation Coefficients (ICC, single measures, absolute agreement) with $95 \%$ confidence intervals and limits of agreement (LoA). Time spent across acceleration levels in $40 \mathrm{mg}$ resolution was also examined. There were no significant differences between the non-dominant and dominant wrist for ENMO, wear time, MVPA or MVPA 10 min. Agreement between wrists were strong for most outcomes (ICC $\geq 0.92$ ) including wear time, ENMO, MVPA, MVPA $_{10 \min }$ and the distribution of time across acceleration levels. Agreement was strong in the low acceleration bands (ICC $=$ 0.970 and 0.922 ) with a mean bias of 3.08 minutes (LoA -55.18 to 61.34$)$ and -5.43 (LoA 43.47 to 32.62 ). In summary, ENMO, MVPA, MVPA $_{10 \mathrm{~min}}$, wear time and the distribution of time across acceleration levels compared well at the group level. The LOA from the two lowest acceleration levels suggest further work over a longer monitoring period is needed to determine whether outputs from each wrist are comparable.

Keywords: GGIR, ENMO, wrist-worn, adults, agreement, MVPA. 
Introduction:

The accurate assessment of physical activity (PA) is important for assessing the effectiveness of public health initiatives aimed at increasing PA and for surveillance purposes. Assessing PA is challenging but accelerometers are increasingly used to quantify PA as they overcome several of the limitations associated with self-report measures (Prince et al., 2008). Of the several different types of accelerometers available (Actical, Actiwatch, GENEActiv, Axivity, etc), the ActiGraph (Pensacola, FL, USA) accelerometers are one of the most commonly used by researchers when measuring PA levels in adults (Wijndaele et al., 2015). Historically accelerometers were worn on the waist to reflect whole body movement and thus energy expenditure but poor compliance and subsequent selection bias and misclassification (Troiano et al., 2014), has seen increased use of wrist-worn accelerometers to assess habitual PA. Wrist-worn accelerometers have also been validated against established measures of physical activity energy expenditure (van Hees et al., 2011; Hildebrand et al., 2014; White et al., 2016) and have shown to provide high (85\%-97\%) activity classification accuracies when using machine learning models (Zhang et al., 2012; Mannini et al., 2013). Despite recent findings which question the classification accuracy of wrist accelerometry processing methods (Ellingson et al., 2017), the use of wrist-worn accelerometers to characterize activity patterns in large cohorts of individuals is now common (Doherty et al., 2017; Menai et al., 2017; NHANES, 2018).

Indeed, wrist-worn accelerometers are being used to assess PA in large population surveys such as the UK Biobank study (Doherty et al., 2017), the Whitehall II cohort (Sabia et al., 2014; Menai et al., 2017) and the US National Health and Nutrition Examination Survey (NHANES) (NHANES, 2018) which used the ActiGraph GT3X+ model in the 2011-2012 and 2013-2014 cycles. In these studies different methodologies have been applied where 
either the non-dominant (Sabia et al., 2015; Menai et al., 2017; NHANES, 2018) or dominant wrist (Doherty et al., 2017) has been used.

The selection of the non-dominant wrist for the NHANES protocol was predicated on historical precedent for sleep research and early wrist accelerometer calibration studies that did not appear to favour the dominant or non-dominant wrist for PA monitoring (Troiano et al., 2014). As these early wrist accelerometer calibration studies were undertaken using the GENEActiv accelerometer however (Esliger et al., 2011; Zhang et al., 2012; Phillips et $a l ., 2013)$, more recent studies using non-proprietary accelerations have suggested that activity estimates may not be equivalent between different brands of accelerometers (Hildebrand et al., 2014; Rowlands et al., 2015). As the dominant arm tends to be stronger and used more often than the non-dominant arm for normal lifestyle activities, Dieu and colleagues (Dieu et al., 2017) recently evaluated the differences in activity outputs from ActiGraph GT3X devices worn simultaneously on the dominant and non-dominant wrist. Here the authors assessed PA using counts produced from proprietary algorithms (Troiano et al., 2014) and reported no significant differences in PA estimates over several axis. As the authors contend nonetheless, their findings are limited since their analysis was only undertaken on total PA (counts. $\mathrm{min}^{-1}$ ) and its possible that their findings would be different if activity outputs were assessed across a range of activity intensities.

An important technological advancement in accelerometry has seen high-resolution raw accelerometry data become available on various devices, including the ActiGraph GT3X+, which allows for the raw data to be processed. When processing raw data, open-source resources such as the GGIR package in R [http:/cran.r-prouect.org] are being used to undertake post-data processing affording greater transparency and consistency of methodologies whilst enhancing comparability between studies using different brands of accelerometer. 
As processing data using GGIR provides researchers with an extremely rich dataset, there is an opportunity to build upon the work previously undertaken by Dieu and colleagues (Dieu et al., 2017) and establish whether activity outputs are comparable across a range of activity intensities when collected from dominant and non-dominant wrist-worn accelerometers. Not only would this allow for the future pooling of accelerometer data that has been collected from different wrist locations, but researchers would not need to instruct and remind study participants to wear the accelerometer device on a specific wrist.

As wrist-worn accelerometers are currently being deployed in large scale studies, their use is likely to increase given their comfort over the traditional hip placement and enhanced compliance rates. As greater compliance rates will provide the researcher with activity data over more days and capture a greater proportion of that day, researchers will have more confidence that the data collected is representative of habitual PA. Recent findings from studies using wrist-worn accelerometers suggest that associations between MVPA with successful ageing (Menai et al., 2017) and adiposity markers (Sabia et al., 2015) is more pronounced when compared to questionnaires. These findings will provide confidence to researchers given the well-established relationships between physical activity and successful ageing (Dogra \& Stathokostas, 2012; Almeida et al., 2014) and adiposity markers (Jensen et al., 2014). As will recent findings which showed that wrist-worn accelerometers is highly acceptable to participants with a median wear-time of 6.9 days and the very high proportion of people $(103,578$ of 106,053 (98\%)) in whom the data were of high quality and completeness (Doherty et al., 2017).

Although it could be argued that similar wear time could be elicited from hip-worn accelerometers (Tudor-Locke et al., 2015), it is important to note that this study was undertaken in children and involved several compliance enhancing strategies including phone calls, daily visits to participants schools as well as small daily incentives (e.g., 
erasers, stickers). Whether the average wear time of 22.6 hours therefore was due to the 24-hour waist worn protocol used or the incentives or compliance strategies used is unclear. Regardless of the reason(s), the increased cost and burden of employing a similar approach to that of Tudor-Locke and colleagues to increase compliance would be challenging and is likely the reason that more attention is being given to the use of wristworn accelerometers to capture PA.

Understanding the agreement between outcomes from the two wrist placement sites across a range of outcome variables and activity intensities will provide researchers with the necessary evidence to determine how comparable outcomes are between wrist locations. Therefore, the purpose of this study was to evaluate the agreement between several activity measures using raw acceleration data from ActiGraph GT3X+ accelerometers worn concurrently on the dominant and non-dominant wrist when processed using GGIR. Outcome variables included measures of ENMO, MVPA, MVPA 10 min, wear time and the distribution of time across acceleration levels.

Methods:

Participants

A convenience sample of 56 adults were recruited from South Lanarkshire. Upon receipt of approval from the ethical committee of the University of the West of Scotland, participants provided written informed consent prior to their participation. Data was collected between October 2017 and December 2017.

Each participant wore two ActiGraph GT3X+ monitors, one on their non-dominant wrist and the other on their dominant wrist. Prior to distribution, both accelerometers were synchronised with Greenwich Mean time and initialized to capture data at $90 \mathrm{~Hz}$. Verbal confirmation of participants non-dominant wrist was provided prior to being instructed to 
wear both devices for a minimum of 8 hours apart from water-based activities. Both accelerometers were set to commence data collection immediately after distribution.

\section{Data Management}

Upon the return of both devices, data were uploaded using ActiLife v6.13.3 (Actigraph, Pensacola, FL, USA) and saved in raw format as GT3X+ files. The GT3X+ files were subsequently converted to csv files containing $\mathrm{x}, \mathrm{y}$ and $\mathrm{z}$ vectors to facilitate raw data processing. Data were then processed in R (http://cran.r-project.org) using the GGIR package (version 1.5-10) which allows raw accelerations (gravitational acceleration) to be processed and analysed (Van Hees et al., 2014). Briefly, the package auto calibrates the raw triaxial accelerometer signals and converts them into one omnidirectional measure of acceleration, termed the signal vector magnitude (SVM). SVM represents the value of gravity (i.e., $\left.\mathrm{SVM}=\sqrt{\left(x^{2}+y^{2}+z^{2}\right)}-1\right)$, with negative values rounded to zero. This metric is referred to as the Euclidean Norm Minus One (ENMO) (van Hees et al., 2013). Raw data were further reduced by calculating the average ENMO values per $5 \mathrm{~s}$ epoch expressed in mg over the monitoring period.

Files were excluded from all analyses if post-calibration error was greater than $0.02 \mathrm{~g}$ or fewer than $8 \mathrm{~h}$ of wear time were recorded by either accelerometer during the monitoring period. Raw data wear times were estimated on the basis of the SD and value range of each axis, calculated for 60 min windows with 15-min moving increments as described in detail elsewhere (van Hees et al., 2013). Briefly, if for at least 2 out of the 3 axes the value range is less than $50 \mathrm{mg}$ or the SD is less than $13 \mathrm{mg}$ the time window is classified as non-wear as reported elsewhere (Rowlands et al., 2016).

ENMO, time in MVPA and the time spent in $\mathrm{MVPA}_{10 \mathrm{~min}}$ were calculated across the monitoring period. To calculate MVPA we used the device specific prediction equations 
provided by Hildebrand and colleagues (Hildebrand et al., 2014) to generate the intensity specific cut-point of $100.6 \mathrm{mg}$. Bouts were identified as $10 \mathrm{~min}$ of consecutive $5 \mathrm{~s}$ epochs whereby $80 \%$ of the epochs were either equal, or higher, than the $100 \mathrm{mg}$ threshold (Rowlands et al., 2016). We also examined the distribution of time spent across acceleration levels in $40 \mathrm{mg}$ resolution (0-40 mg, 40-80 mg, 80-120 mg....>400 mg)) which were calculated from $6 \mathrm{am}-11 \mathrm{pm}$.

Statistical Analysis

Descriptive statistics (mean \pm SD) were calculated for summary outcome variables. Histograms, Q-Q plots and Kolmogorov-Smirnov tests were used to confirm the normal distribution of the summary outcome variables. Thereafter, paired t-tests were undertaken with sequential Holm-Bonferroni corrections (Holm, 1979). Level of agreement between outcome variables from the dominant and non-dominant wrists was examined using Intraclass Correlation Coefficients (ICC, single measures, absolute agreement) with $95 \%$ confidence intervals and limits of agreement (LoA) (Bland \& Altman, 1986). All statistical analysis was undertaken using IBM SPSS Statistics v24. Alpha was set at 0.05.

Results:

One participant experienced a device malfunction (reason unknown) with their accelerometer and were subsequently excluded from the analysis. No data files were excluded based on calibration error. All the remaining 55 participants (29 females, age: $31.9 \pm 9.7$ years; height: $1.65 \pm 9.3 \mathrm{~m}$; mass: $76.7 \pm 16.5 \mathrm{~kg}$ ) wore both accelerometer devices for a minimum of 8 hours and were included within the analysis.

There were no significant differences in values between the non-dominant and dominant wrist for ENMO, wear time, MVPA or MVPA10min (Table 1). Agreement between the dominant and non-dominant wrist locations (Table 2) was strong for the majority of 
outcomes (ICC $\geq 0.92)$ including wear time, ENMO, MVPA, MVPA $10 \min$ and the distribution of time across acceleration levels. Agreement was particularly strong in the low acceleration bands where most of the time was spent in by participants (Table 2). Mean biases were low and tended to be positive for the distribution of time across acceleration levels (Table 2). The dominant wrist placement captured more minutes in the lowest acceleration level whereas the non-dominant wrist captured more minutes in the $2^{\text {nd }}$ lowest acceleration level. No significant differences were evident for the distribution of time across acceleration levels (Figure 1).

Discussion:

The purpose of this study was to evaluate the agreement between several activity measures using raw acceleration data from ActiGraph GT3X+ accelerometers worn concurrently on the dominant and non-dominant wrist when processed using GGIR. Our findings revealed a high agreement between the variables produced from GGIR when comparing outputs from the dominant and non-dominant wrists. When examining ENMO, MVPA, MVPA $_{10 \mathrm{~min}}$, wear time and the distribution of time across acceleration levels the outputs were comparable. To the best of our knowledge, this is the first study to compare several activity measures using raw acceleration data from ActiGraph GT3X+ accelerometers worn concurrently on the dominant and non-dominant wrist when processed using GGIR.

As time spent in MVPA is often used to quantify the number of adults meeting current PA recommendations (World Health Organization, 2010; Department of Health, 2016; US Department of Health and Human Services, 2018), it is encouraging to note the strong agreement in this outcome when captured from the dominant and non-dominant wrist locations. There was also strong agreement for MVPA accumulated in 10-min bouts. The limits of agreement however for both outcomes were wide. Rowlands and colleagues 
(Rowlands et al., 2016) demonstrated similar findings when comparing the activity outputs from GGIR when subjects wore GENEActiv and ActiGraph accelerometers on their nondominant wrists although in contrast to this study, the authors reported a small limit of agreement for MVPA accumulated in 10-min bouts. Given the differences in wrist placements between studies it is difficult to make direct comparisons between findings but a likely explanation for the wide limits of agreement could be attributed to a decoupling effect (Rowlands et al., 2014; Fairclough et al., 2016; Noonan et al., 2017). For instance, wrist accelerations may be higher for the dominant wrist when undertaking activities such as writing, fidgeting and eating with a single utensil in comparison to the non-dominant wrist.

There were trivial differences in ENMO when compared between the dominant and nondominant wrists suggesting a strong agreement between wrist locations. The limits of agreement however were wide. This is an important finding and suggests that ENMO could be used to rank participants by activity level at a group level and could distinguish active from non-active participants, regardless of wrist placement. Nonetheless, caution is advised when making such interpretations at the individual level given the wide limits of agreement. The greatest differences in time spent across acceleration levels between wrist locations were found in the two lowest acceleration levels (Table 2). It has been proposed that the commonly used threshold of 100 counts. minute ${ }^{-1}$ to determine time in sedentary behaviour equates to an acceleration threshold of approximately $50 \mathrm{mg}$ (Rowlands et al., 2016). Estimates of time spent in the $0-40 \mathrm{mg}$ acceleration level differed by $3 \mathrm{mins}$ with the dominant wrist recording more minutes than the non-dominant wrist whereas time spent in the $40-80 \mathrm{mg}$ acceleration level differed by $5.5 \mathrm{mins}$ with the non-dominant wrist recording more minutes than the dominant wrist. 
More minutes were recorded from the dominant wrist across most of the acceleration levels. Even though more minutes were recorded from the non-dominant wrist within the 40-80 mg acceleration level, the lack of significant differences in average ENMO output between wrist locations is likely the result of the dominant wrist recording more time spent in the other acceleration levels. As the majority of participants spend their time in sedentary and light activities over the course of a day however (da Silva et al., 2014; Rowlands et al., 2016), these small differences in accelerations from one day could be greater over a longer monitoring period.

To measure sedentary behaviour, it is necessary to measure posture (sitting or reclining) as well as energy expenditure (Tremblay et al., 2010). At present, devices such as the ActiGraph GT3X+ can be used to estimate sedentary behaviour but they do this based on minimal or no movement. A situation where this could lead to the misclassification of activity is during writing activities. For instance, if a participant is writing, the accelerometer placed on the writing wrist would likely capture accelerations and classify activity as light, moderate or vigorous whereas the other accelerometer placed on the nonwriting wrist which is typically stationary, captures minimal accelerations and may classify activity as sedentary. It is unclear the nature of activities participants was undertaking throughout the monitoring period but it's unlikely that activities requiring the use of only one wrist was extensive. Moreover, the short monitoring period may not accurately reflect the true physical activity and sedentary behaviour patterns of participants and further work over a longer monitoring period may be necessary to uncover these differences.

It has recently been proposed that that the ActiGraph GT3X accelerometer worn on the dominant or non-dominant wrist assesses PA similarly and that the results from previous studies are comparable, regardless of the wrist used (Dieu et al., 2017). The authors note 
several limitations within their study but there are additional concerns we have identified which questions the suitability of this recommendation. In their analysis (Dieu et al., 2017) only counts. minute ${ }^{-1}$ was used to provide a measure of total physical activity, despite the availability of published adult thresholds for Actigraph wrist-worn accelerometers (Hildebrand et al., 2014). Although we acknowledge that measures of total PA are often used in studies to quantify activity levels, we feel it is premature to conclude there is no difference in PA assessment between an accelerometer worn on the dominant or nondominant wrist based on one activity metric. Another concern relates to the methods used to assess the comparability of the outputs from the dominant and non-dominant wrist. Dieu and colleagues relied upon paired Student's t-tests and Pearson correlations but correlation examines the relationship between one variable and another whereas paired t-tests detects the mean difference between two groups. Neither test is appropriate when trying to assess the degree of agreement and comparability between two measures (Giavarina, 2015). Without the use and interpretation of Bland Altman Analysis (Bland \& Altman, 1986), it is difficult to say with certainty whether outputs from the dominant and non-dominant wrist are comparable from the work undertaken by Dieu and colleagues.

Strengths of this study include the use of a popular accelerometer and the use of open source software to run GGIR and process the data. Given the advantages of processing raw data in GGIR which allows for a more advanced analysis of several activity measures, control over data processing and increased transparency, future work processing data in GGIR should be undertaken. The homogenous sample and short data collection period used is a limitation of this study. Future work should consider a longer data collection period to capture a wider range of activity and sedentary behaviour which may accurately reflect habitual patterns. 
In summary, there was a high agreement between ENMO, MVPA, MVPA ${ }_{10 m i n}$, wear time and the distribution of time across acceleration levels outputs from the dominant and nondominant wrists. Wear time output was identical between the two devices whereas the bias for ENMO, MVPA and MVPA10min was small. The 95\% limits of agreement for ENMO, MVPA and MVPA $10 \min$ were wide however suggesting that researchers should exert a degree of caution if interested in quantifying these measures from an accelerometer placed on an alternative wrist to that where published thresholds or values were derived from. There was a high agreement between time spent across acceleration levels with the greatest differences between wrist locations found in the two lowest acceleration levels. Although the bias was small, the $95 \%$ limits of agreement suggest that over a longer data collection period, these could become wide. Given these findings, researchers should exert caution when comparing the magnitude of overall physical activity through ENMO between wrist placements until further work over a longer data collection period is undertaken.

Acknowledgements:

This study was partly funded by The University of the West of Scotland, VP Research Fund. This research did not receive any specific grant from funding agencies in the public, commercial, or not-for-profit sectors.

Conflict of interest:

The authors declare they have no conflict of interest. 
References:

Almeida OP, Khan KM, Hankey GJ, Yeap BB, Golledge J \& Flicker L (2014). 150 minutes of vigorous physical activity per week predicts survival and successful ageing: a population-based 11-year longitudinal study of 12201 older Australian men. Br J Sports Med 48, 220-225.

Bland JM \& Altman DG (1986). Statistical methods for assessing agreement between two methods of clinical measurement. Lancet Lond Engl 1, 307-310.

Department of Health (2016). Childhood obesity: a plan for action - GOV.UK. Available at: https://www.gov.uk/government/publications/childhood-obesity-a-plan-foraction [Accessed September 11, 2017].

Dieu O, Mikulovic J, Fardy PS, Bui-Xuan G, Béghin L \& Vanhelst J (2017). Physical activity using wrist-worn accelerometers: comparison of dominant and nondominant wrist. Clin Physiol Funct Imaging 37, 525-529.

Dogra S \& Stathokostas L (2012). Sedentary behavior and physical activity are independent predictors of successful aging in middle-aged and older adults. $J$ Aging Res 2012, 190654.

Doherty A, Jackson D, Hammerla N, Plötz T, Olivier P, Granat MH, White T, Hees VT van, Trenell MI, Owen CG, Preece SJ, Gillions R, Sheard S, Peakman T, Brage S \& Wareham NJ (2017). Large Scale Population Assessment of Physical Activity Using Wrist Worn Accelerometers: The UK Biobank Study. PLOS ONE 12, e0169649.

Ellingson LD, Hibbing PR, Kim Y, Frey-Law LA, Saint-Maurice PF \& Welk GJ (2017). Lab-based validation of different data processing methods for wrist-worn ActiGraph accelerometers in young adults. Physiol Meas 38, 1045.

Esliger DW, Rowlands AV, Hurst TL, Catt M, Murray P \& Eston RG (2011). Validation of the GENEA Accelerometer. Med Sci Sports Exerc 43, 1085-1093.

Fairclough SJ, Noonan R, Rowlands AV, Van Hees V, Knowles Z \& Boddy LM (2016). Wear Compliance and Activity in Children Wearing Wrist- and Hip-Mounted Accelerometers. Med Sci Sports Exerc 48, 245-253.

Giavarina D (2015). Understanding Bland Altman analysis. Biochem Medica Biochem Medica 25, 141-151.

van Hees VT, Gorzelniak L, Dean Leon EC, Eder M, Pias M, Taherian S, Ekelund U, Renstrom F, Franks PW, Horsch A \& Brage S (2013). Separating movement and gravity components in an acceleration signal and implications for the assessment of human daily physical activity. PLoS One 8, e61691.

van Hees VT, Renstrom F, Wright A, Gradmark A, Catt M, Chen KY, Lof M, Bluck L, Pomeroy J, Wareham NJ, Ekelund U, Brage S \& Franks PW (2011). Estimation of daily energy expenditure in pregnant and non-pregnant women using a wrist-worn tri-axial accelerometer. PLOS ONE 6, e22922. 
Hildebrand M, Van Hees V, Hansen BH \& Ekelund U (2014). Age group comparability of raw accelerometer output from wrist- and hip-worn monitors. Med Sci Sports Exerc 46, $1816-1824$.

Holm S (1979). A Simple Sequentially Rejective Multiple Test Procedure. Scand J Stat 6, 65-70.

Jensen MD, Ryan DH, Apovian CM, Ard JD, Comuzzie AG, Donato KA, Hu FB, Hubbard VS, Jakicic JM, Kushner RF, Loria CM, Millen BE, Nonas CA, PiSunyer FX, Stevens J, Stevens VJ, Wadden TA, Wolfe BM \& Yanovski SZ (2014). 2013 AHA/ACC/TOS Guideline for the Management of Overweight and Obesity in Adults: A Report of the American College of Cardiology/American Heart Association Task Force on Practice Guidelines and The Obesity Society. J Am Coll Cardiol 63, 2985-3023.

Mannini A, Intille SS, Rosenberger M, Sabatini AM \& Haskell W (2013). Activity recognition using a single accelerometer placed at the wrist or ankle. Med Sci Sports Exerc 45, 2193-2203.

Menai M, Hees VT van, Elbaz A, Kivimaki M, Singh-Manoux A \& Sabia S (2017). Accelerometer assessed moderate-to-vigorous physical activity and successful ageing: results from the Whitehall II study. Sci Rep 7, 45772.

NHANES (2018). NHANES - National Health and Nutrition Examination Survey Homepage. Available at: https://www.cdc.gov/nchs/nhanes/index.htm [Accessed February 26, 2018].

Noonan RJ, Boddy LM, Kim Y, Knowles ZR \& Fairclough SJ (2017). Comparison of children's free-living physical activity derived from wrist and hip raw accelerations during the segmented week. J Sports Sci 35, 2067-2072.

Phillips LRS, Parfitt G \& Rowlands AV (2013). Calibration of the GENEA accelerometer for assessment of physical activity intensity in children. J Sci Med Sport 16, 124128.

Prince SA, Adamo KB, Hamel ME, Hardt J, Gorber SC \& Tremblay M (2008). A comparison of direct versus self-report measures for assessing physical activity in adults: a systematic review. Int J Behav Nutr Phys Act 5, 56.

Rowlands AV, Fraysse F, Catt M, Stiles VH, Stanley RM, Eston RG \& Olds TS (2015). Comparability of measured acceleration from accelerometry-based activity monitors. Med Sci Sports Exerc 47, 201-210.

Rowlands AV, Rennie K, Kozarski R, Stanley RM, Eston RG, Parfitt GC \& Olds TS (2014). Children's physical activity assessed with wrist- and hip-worn accelerometers. Med Sci Sports Exerc 46, 2308-2316.

Rowlands AV, Yates T, Davies M, Khunti K \& Edwardson CL (2016). Raw Accelerometer Data Analysis with GGIR R-package: Does Accelerometer Brand Matter? Med Sci Sports Exerc 48, 1935-1941. 
Sabia S, Cogranne P, Hees VT van, Bell JA, Elbaz A, Kivimaki M \& Singh-Manoux A (2015). Physical Activity and Adiposity Markers at Older Ages: Accelerometer Vs Questionnaire Data. J Am Med Dir Assoc 16, 438.e7-438.e13.

Sabia S, Hees V, T V, Shipley MJ, Trenell MI, Hagger-Johnson G, Elbaz A, Kivimaki M \& Singh-Manoux A (2014). Association Between Questionnaire- and Accelerometer-Assessed Physical Activity: The Role of Sociodemographic Factors. Am J Epidemiol 179, 781-790.

da Silva IC, van Hees VT, Ramires VV, Knuth AG, Bielemann RM, Ekelund U, Brage S \& Hallal PC (2014). Physical activity levels in three Brazilian birth cohorts as assessed with raw triaxial wrist accelerometry. Int J Epidemiol 43, 1959-1968.

Tremblay MS, Colley RC, Saunders TJ, Healy GN \& Owen N (2010). Physiological and health implications of a sedentary lifestyle. Appl Physiol Nutr Metab 35, 725-740.

Troiano RP, McClain JJ, Brychta RJ \& Chen KY (2014). Evolution of accelerometer methods for physical activity research. Br J Sports Med 48, 1019-1023.

Tudor-Locke C et al. (2015). Improving wear time compliance with a 24-hour waist-worn accelerometer protocol in the International Study of Childhood Obesity, Lifestyle and the Environment (ISCOLE). Int J Behav Nutr Phys Act 12, 11.

US Department of Health and Human Services (2018). Physical Activity Guidlines for Americans. Advisory Committee report 2018. Available from https://health.gov/paguidelines/second-edition/report.aspx. Accessed 7th March 2018. Washington DC. Available at: https://health.gov/paguidelines/secondedition/report.aspx.

Van Hees VT, Fang Z, Langford J, Assah F, Mohammad A, Silva ICM da, Trenell MI, White T, Wareham NJ \& Brage S (2014). Autocalibration of accelerometer data for free-living physical activity assessment using local gravity and temperature: an evaluation on four continents. J Appl Physiol 117, 738-744.

White T, Westgate K, Wareham NJ \& Brage S (2016). Estimation of Physical Activity Energy Expenditure during Free-Living from Wrist Accelerometry in UK Adults. PLOS ONE 11, e 0167472.

Wijndaele K, Westgate KL, Stephens SK, Blair SN, Bull FC, Chastin SF., Dunstan DW, Ekelund U, Esliger DW, Freedson PS, Granat MH, Matthews CE, Owen N, Rowlands AV, Sherar LB, Tremblay MS, Troiano R, Brage S \& Healy GN (2015). Utilization and Harmonization of Adult Accelerometry Data: Review and Expert Consensus. Med Sci Sports Exerc 47, 2129-2139.

World Health Organization (2010). Global Recommendations on Physical Activity for Health. Available from http://whqlibdoc.who.int/publications/2010/9789241599979_eng.pdf. Accessed 8th August 2011. World Health Organization, Geneva.

Zhang S, Rowlands AV, Murray P \& Hurst TL (2012). Physical activity classification using the GENEA wrist-worn accelerometer. Med Sci Sports Exerc 44, 742-748. 
Table 1. Summary GGIR outcome variables from acceleration measured at the wrist by the Actigraph GT3X+

\begin{tabular}{lcc}
\hline & Dominant & Non \\
\hline Wear time (h) & $11.99 \pm 3.67$ & $11.99 \pm 3.67$ \\
ENMO (mg) & $17.96 \pm 11.17$ & $18.01 \pm 11.64$ \\
MVPA (min) & $75.06 \pm 57.12$ & $72.92 \pm 56.09$ \\
MVPA $_{10 \min }$ & $5.42 \pm 11.86$ & $8.01 \pm 16.52$ \\
\hline
\end{tabular}


Table 2. Agreement between GGIR outcome variables from ActiGraph GT3X+ accelerometers worn on the dominant and non-dominant wrists.

\begin{tabular}{lccc}
\hline & ICC $(95 \% \mathrm{CI})$ & $\begin{array}{c}\text { Mean bias }(95 \% \\
\text { LoA } *\end{array}$ & Range of values** \\
\hline Wear time (h) & $1.00(1.00,1.00)$ & $0(0,0)$ & $8-18$ \\
\hline Overall activity $(\mathrm{mg})$ & & & \\
ENMO (mg) & $0.964(0.938,0.979)$ & $-0.05(-6.14,6.04)$ & $2.98-48.81$ \\
\hline MVPA (min) & & & \\
MVPA & $0.972(0.952,0.983)$ & $2.15(-24.24,28.53)$ & $4.30-216.55$ \\
MVPA $10 \mathrm{~min}$ & $0.955(0.924,0.973)$ & $0.27(-18.48,19.02)$ & $0-142.55$ \\
\hline Distribution of time across acceleration levels $(\mathrm{min}, 6.00-23.00)$ & \\
$0-40 \mathrm{mg}$ & $0.970(0.949,0.982)$ & $3.08(-55.18,61.34)$ & $609.42-1017.63$ \\
$40-80 \mathrm{mg}$ & $0.922(0.868,0.954)$ & $-5.43(-43.47,32.62)$ & $1.21-182.21$ \\
$80-120 \mathrm{mg}$ & $0.964(0.939,0.979)$ & $0.68(-15.32,16.68)$ & $0.71-110.63$ \\
$120-160 \mathrm{mg}$ & $0.940(0.900,0.965)$ & $-0.02(-13.05,12.57)$ & $0.25-68.13$ \\
$160-200 \mathrm{mg}$ & $0.954(0.922,0.973)$ & $0.53(-6.04,7.09)$ & $0.17-40.33$ \\
$200-240 \mathrm{mg}$ & $0.954(0.920,0.973)$ & $0.51(-3.18,4.21)$ & $0-23.34$ \\
$240-280 \mathrm{mg}$ & $0.933(0.887,0.960)$ & $0.32(-2.34,2.99)$ & $0-17.05$ \\
$280-320 \mathrm{mg}$ & $0.856(0.762,0.914)$ & $0.33(-1.95,2.61)$ & $0-10.30$ \\
$320-360 \mathrm{mg}$ & $0.927(0.878,0.957)$ & $0.07(-1.27,1.40)$ & $0-9.75$ \\
$360-400 \mathrm{mg}$ & $0.817(0.706,0.889)$ & $0.04(-1.44,1.52)$ & $0-6.34$ \\
$>400 \mathrm{mg}$ & $0.840(0.741,0.904)$ & $0.11(-5.54,5.76)$ & $0-28.92$ \\
\hline
\end{tabular}

* Comparisons always made between the dominant vs. non-dominant wrist. **Range of values were calculated as the mean minimum and mean maximum from the dominant and non-dominant wrists. 


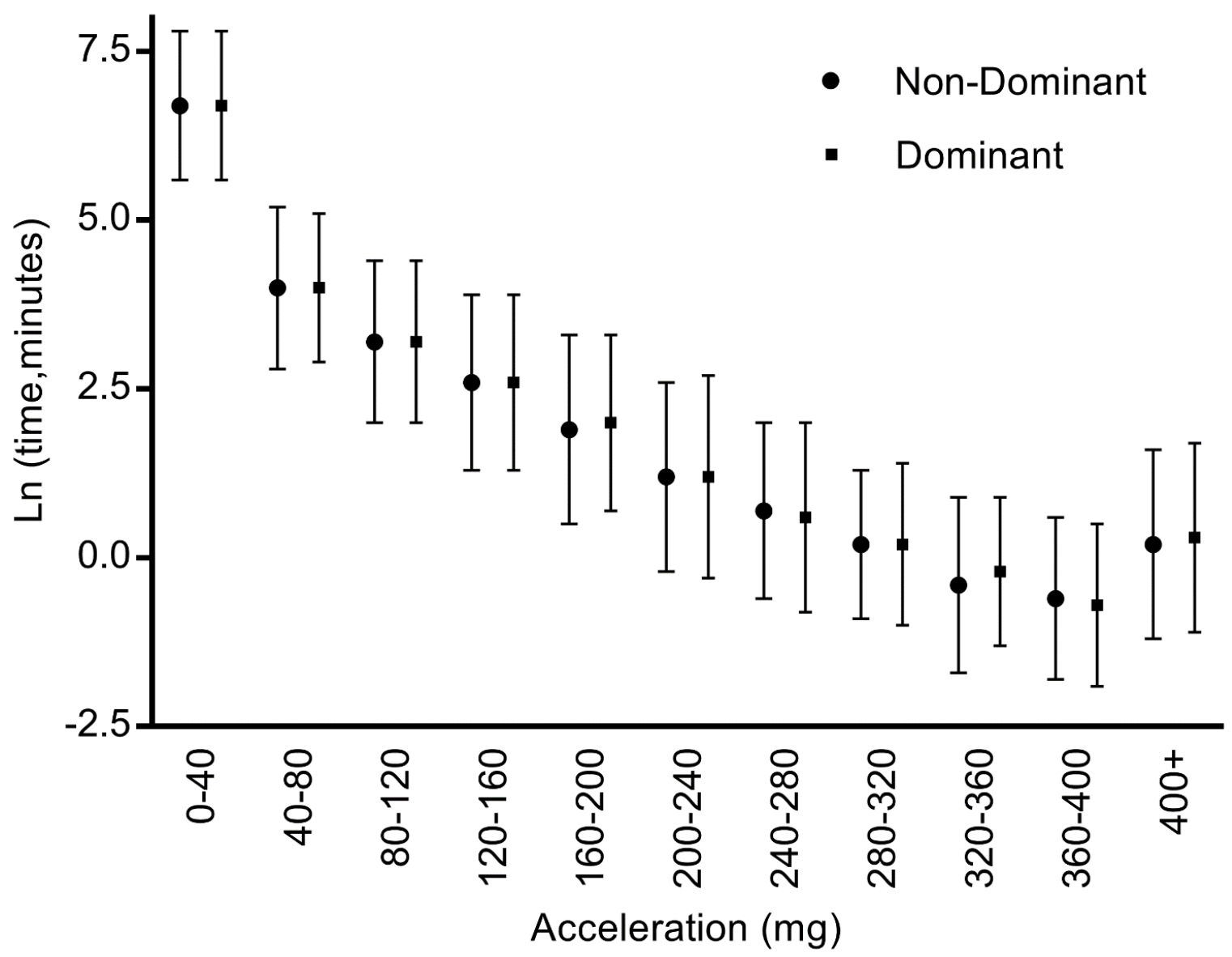

Figure 1. Distribution of time across acceleration levels in 40mg categories measured by the ActiGraph GT3X+ worn concurrently on the dominant and non-dominant wrists $(6 \mathrm{am}-11 \mathrm{pm})$. The natural log of time in minutes is plotted on the y-axis. 